\title{
NONLINEAR EIGENVALUE PROBLEMS WITH SPECIFIED EIGENVALUES*
}

\author{
MICHAEL KAROW ${ }^{\dagger}$, DANIEL KRESSNER ${ }^{\ddagger}$, AND EMRE MENGI ${ }^{\S}$
}

\begin{abstract}
This work considers eigenvalue problems that are nonlinear in the eigenvalue parameter. Given such a nonlinear eigenvalue problem $T$, we are concerned with finding the minimal backward error such that $T$ has a set of prescribed eigenvalues with prescribed algebraic multiplicities. We consider backward errors that only allow constant perturbations, which do not depend on the eigenvalue parameter. While the usual resolvent norm addresses this question for a single eigenvalue of multiplicity one, the general setting involving several eigenvalues is significantly more difficult. Under mild assumptions, we derive a singular value optimization characterization for the minimal perturbation that addresses the general case.
\end{abstract}

Key words. nonlinear eigenvalue problem, analytic matrix-valued function, Sylvester-like operator, backward error

AMS subject classifications. 65F $15,65 \mathrm{~F} 18,47 \mathrm{~A} 56$

DOI. $10.1137 / 130927462$

1. Introduction. Let $T: \Omega \rightarrow \mathbb{C}^{n \times n}$ be an analytic matrix-valued function on some domain $\Omega \subset \mathbb{C}$. Then $\lambda \in \mathbb{C}$ is called an eigenvalue of $T$ if there is a nonzero vector $v \in \mathbb{C}^{n}$ such that

$$
T(\lambda) v=0 .
$$

A number of numerical algorithms have been proposed for solving such nonlinear eigenvalue problems; see [25, 32] for overviews. In these algorithms, it is important to be able to quantify whether the accuracy of the current eigenvalue approximation $\mu \in \Omega$ is satisfactory. Let us first suppose that an eigenvector approximation $\tilde{v} \in \mathbb{C}^{n}$, normalized such that $\|\tilde{v}\|_{2}=1$, is also at hand. Then the norm of the residual,

$$
\|T(\mu) \tilde{v}\|_{2},
$$

is the most common way for quantifying the accuracy of $\mu$ and $\tilde{v}$ together. In the absence of eigenvector information or for testing the backward error of the eigenvalue approximation $\mu$ alone, one is free to choose a normalized vector $\tilde{v}$ such that (1.2) is minimized. This leads to

$$
\sigma_{\min }(T(\mu))=\left\|T(\mu)^{-1}\right\|_{2}^{-1},
$$

where $\sigma_{\min }$ denotes the smallest singular value of a matrix. For a linear eigenvalue problem $T(\lambda)=\lambda I-A$, the quantity (1.3) corresponds to the well-established notion

\footnotetext{
* Received by the editors July 2, 2013; accepted for publication (in revised form) by F. Tisseur April 22, 2014; published electronically July 1, 2014.

http://www.siam.org/journals/simax/35-3/92746.html

${ }^{\dagger}$ Department of Mathematics, TU-Berlin, Sekretariat MA 4-5, Strasse des 17. Juni 136, D-10623, Berlin, Germany (karow@math.tu-berlin.de).

†SB-MATHICSE-ANCHP, EPF Lausanne, Station 8, CH-1015 Lausanne, Switzerland (daniel. kressner@epfl.ch).

$\S$ Department of Mathematics, Koç University, Rumelifeneri Yolu, 34450 Sarıyer-İstanbul, Turkey (emengi@ku.edu.tr). The work of this author was supported in part by the European Commision grant PIRG-GA-268355, the TÜBİTAK (the Scientific and Technological Research Council of Turkey) carrier grant 109T660, and by the BAGEP program of Turkish Academy of Science.
}

819 
of pseudospectra [31]. Different extensions of pseudospectra to the nonlinear case have been proposed in $[8,28,30,33]$.

In applications, one is often interested in approximating more than one eigenvalue. This gives rise to the task of quantifying how well a list of $s$ (not necessarily distinct) scalars $\lambda_{1}, \ldots, \lambda_{s} \in \Omega$ approximates eigenvalues of $T$. Of course, one could apply (1.3) to each eigenvalue approximation individually, but this bears the danger of failing to detect spurious eigenvalue approximations. More specifically, consider the case of two eigenvalue approximations $\lambda_{1}, \lambda_{2}$, both with small backward errors based on the quantity (1.3), but $\lambda_{1}$ and $\lambda_{2}$ actually approximate the same (simple) eigenvalue. Then $\lambda_{1}, \lambda_{2}$ taken together may be a poor approximation to two eigenvalues of $T$. In this paper, we propose and analyze an approach that is robust to this phenomenon. As explained in section 6 , the use of this approach goes beyond verifying the backward errors of eigenvalue approximations. For example, it can be used to determine the distance to a nearest nonlinear eigenvalue problem with a multiple eigenvalue.

Based on the well-known characterization of $\sigma_{\min }(T(\mu))$ as the minimal $\|\Delta\|_{2}$ among all $\Delta \in \mathbb{C}^{n \times n}$ such that $T(\mu)+\Delta$ is singular, we propose the distance

$$
\tau_{r}(\mathbb{S}):=\min \left\{\|\Delta\|_{2} \mid \sum_{j=1}^{s} m_{j}(T+\Delta) \geq r\right\},
$$

where $\mathbb{S}:=\left\{\lambda_{1}, \ldots, \lambda_{s}\right\} \subset \Omega, r$ is a specified positive integer, and $m_{j}(T+\Delta)$ denotes the algebraic multiplicity of $\lambda_{j}$ as an eigenvalue of $\widetilde{T}(\lambda)=T(\lambda)+\Delta$. With the definition above, we require that $r$ eigenvalues of $\widetilde{T}(\lambda)$, counting their algebraic multiplicities, belong to $\mathbb{S}$, but it is possible that $m_{j}(T+\Delta)=0$ for some $j$; thus $\lambda_{j} \in \mathbb{S}$ may possibly not be an eigenvalue of $\widetilde{T}(\lambda)$. Note that the algebraic multiplicity of $\lambda_{j}$ is defined as the multiplicity of $\lambda_{j}$ as a root of the scalar function $\operatorname{det}(\tilde{T}(\lambda))$. Specifically, when $\mathbb{S}=\{\mu\}$ and $r=1$, this distance has the singular value characterization (1.3).

The distance (1.4) extends our previous definitions for (generalized) linear and polynomial eigenvalue problems $[22,19]$ to the nonlinear case. In these earlier works, we derived a singular value optimization characterization for $\tau_{r}(\mathbb{S})$, which can be numerically solved using global optimization techniques, at least when $r$ is small [26]. The main contribution of this work is to show that such a characterization also holds for the nonlinear case. A notable feature of our derivation is that it does not make use of linearization techniques but works directly with the original nonlinear eigenvalue problems, based on the technique of so-called invariant pairs [21].

The rest of this paper is organized as follows. In section 2, we first analyze the nullspace of a linear, Sylvester-like operator associated with an analytic matrixvalued function $T$. This result then yields a rank characterization for $T$ to have sufficiently many eigenvalues belonging to $\mathbb{S}$. Subsequently, in section 3 , we turn this rank characterization into a singular value characterization, which leads to our main result summarized in section 4 . As this result involves the matricization of the Sylvester-like operator, it may be hard to interpret at first. In section 5 , we, therefore, show how our result can be brought into more intuitive form, in terms of the divided differences of $T$. Finally, in section 6 , we illustrate the findings of this paper for two numerical examples. The first example is a small-scale nonlinear eigenvalue problem for which we prescribe a multiple eigenvalue, while the second example is a large-scale problem for which we prescribe two distinct eigenvalues. 
2. A rank characterization for eigenvalues of $\boldsymbol{T}$. For the rest of this paper, we assume that $T: \Omega \rightarrow \mathbb{C}^{n \times n}$ is regular; that is, its determinant $z \mapsto \operatorname{det}(T(z))$ does not vanish identically on $\Omega$. Following the developments in [22, 19], we will approach the distance (1.4) by first characterizing the inequality

$$
\sum_{j=1}^{s} m_{j}(T) \geq r
$$

in terms of the rank of a certain linear operator.

2.1. A linear operator for nonlinear eigenvalue problems. The natural extension of the usual Sylvester operator to the nonlinear setting is most easily defined if we suppose that $T$ takes the form

$$
T(\lambda)=f_{1}(\lambda) A_{1}+f_{2}(\lambda) A_{2}+\cdots+f_{K}(\lambda) A_{K}
$$

for analytic functions $f_{k}: \Omega \rightarrow \mathbb{C}$ and matrices $A_{k} \in \mathbb{C}^{n \times n}, k=1, \ldots, K$. Of course, this is always possible by decomposing $T(\lambda)$ into $n^{2}$ terms $[T(\lambda)]_{i j} e_{i} e_{j}^{T}$, but in many applications $T$ is already given in the form (2.2). For a fixed matrix $C \in \mathbb{C}^{r \times r}$ with all eigenvalues contained in $\Omega$, we define

$$
\mathbb{T}_{C}: \mathbb{C}^{n \times r} \rightarrow \mathbb{C}^{n \times r}, \quad X \mapsto \mathbb{T}_{C}(X):=\sum_{k=1}^{K} A_{k} X f_{k}(C),
$$

where $f_{k}(C)$ is a matrix function in the sense of [18]. Equivalently $[6,7], \mathbb{T}_{C}$ can be defined by the relation

$$
\mathbb{T}_{C}(X)=\frac{1}{2 \pi \mathrm{i}} \int_{\mathcal{C}} T(z) X(z I-C)^{-1} \mathrm{~d} z
$$

where $\mathcal{C}$ is a contour (i.e., a simply closed curve) in $\Omega$ enclosing the eigenvalues of $C$ in its interior. The linear operator $\mathbb{T}_{C}$ is closely associated with the notion of invariant pairs for nonlinear eigenvalue problems [21]. In particular, the nullspace of $\mathbb{T}_{C}$ is trivial if and only if $C$ and $T$ have no eigenvalue in common. To arrive at a rank characterization for (2.1), we need to characterize the nullspace of $\mathbb{T}_{C}$ when $C$ and $T$ do have eigenvalues in common.

First, because of $\mathbb{T}_{C}(X)=\mathbb{T}_{S^{-1} C S}(X S) \cdot S^{-1}$, we may assume without loss of generality that $C$ is in Jordan canonical form:

$$
C=\operatorname{diag}\left(J_{\mu_{1}, r_{1}}, \ldots, J_{\mu_{q}, r_{q}}\right),
$$

where $J_{\mu, r}$ denotes the Jordan block of size $r$ with eigenvalue $\mu$, i.e., $J_{\mu, r}=\mu I+N_{r}$ with

$$
N_{r}=\left[\begin{array}{cccc}
0 & 1 & & \\
& \ddots & \ddots & \\
& & \ddots & 1 \\
& & & 0
\end{array}\right] \in \mathbb{C}^{r \times r} .
$$

For a single Jordan block $J_{\mu, r}$ we will abbreviate $\mathbb{T}_{J_{\mu, r}}$ by $\mathbb{T}_{\mu, r}$. Partitioning $X=$ $\left[X_{1}, \ldots, X_{q}\right]$ with $X_{i} \in \mathbb{C}^{n \times r_{i}}$, it follows from

$$
\mathbb{T}_{C}(X)=\left[\mathbb{T}_{\mu_{1}, r_{1}}\left(X_{1}\right), \ldots, \mathbb{T}_{\mu_{q}, r_{q}}\left(X_{q}\right)\right]
$$


that

$$
\operatorname{dim} \operatorname{ker} \mathbb{T}_{C}=\sum_{i=1}^{q} \operatorname{dim} \operatorname{ker} \mathbb{T}_{\mu_{i}, r_{i}} .
$$

Hence, we can restrict our discussion to linear operators of the type $\mathbb{T}_{\mu, r}$.

2.2. Root polynomials. It is well known $[17,27,5]$ that the equation $\mathbb{T}_{\mu, r}(X)=$ 0 is closely connected to the concept of root polynomials.

A vector polynomial

$$
\phi(z)=\sum_{k=0}^{r} x_{k}(z-\mu)^{k}, \quad x_{k} \in \mathbb{C}^{n},
$$

is called a root polynomial belonging to the eigenvalue $\mu$ of $T$ if $\phi(\mu) \neq 0$ and $T(\mu) \phi(\mu)=0$. Since $z \mapsto \operatorname{det}(T(z))$ does not vanish identically the same holds for the function $z \mapsto T(z) \phi(z)$. Thus, there exists a maximal integer $\kappa \geq 1$, called the order of $\phi$, such that

$$
T(z) \phi(z)=(z-\mu)^{\kappa} \psi(z)
$$

for some analytic vector function $\psi$ defined in a neighborhood of $\mu$ and satisfying $\psi(\mu) \neq 0$. In terms of the derivatives of $T(z) \phi(z)$, we have

$$
\kappa=\max \left\{k \mid(T \phi)^{(j)}(\mu)=0 \text { for } j=0, \ldots, k-1\right\} .
$$

Recall that the multiplicity $m(\mu)$ of the eigenvalue $\mu$ is defined as the multiplicity of $\mu$ as a root of $\operatorname{det}(T(z))$. In contrast, the multiplicity of an eigenvector $x_{0}$ is the maximal possible order of any root polynomial $\phi(z)$ with $\phi(\mu)=x_{0}$.

The following theorem characterizes a particular set of root polynomials.

TheOrem 2.1 (see $[16,27]$ ). Let $\mu$ be an eigenvalue of the regular analytic matrix-valued function $T$. Then there exist root polynomials

$$
\phi_{i}(z)=\sum_{k=0}^{\kappa_{i}-1} x_{i k}(z-\mu)^{k}, \quad i=1, \ldots, s,
$$

to the eigenvalue $\mu$ with orders $\kappa_{1} \geq \cdots \geq \kappa_{s}$ such that the vectors $x_{i 0}=\phi_{i}(\mu), i=$ $1, \ldots, s$, form a basis of $\operatorname{ker} T(\mu)$ and $\sum_{i=1}^{s} \kappa_{i}=m(\mu)$.

A set of root polynomials $\phi_{i}$ having the properties described in Theorem 2.1 is called a canonical system of root polynomials. The orders $\kappa_{i}$ in such a canonical system are unique [27] and are called the partial multiplicities of the eigenvalue $\mu$. Note that the degree of $\phi_{i}$ cannot be larger than $\kappa_{i}-1$, but it can be smaller. Moreover, for each $i=1, \ldots, s$ it holds $[20$, p. 20$]$ that $x_{i 0}$ attains the maximal possible multiplicity among all eigenvectors not expressible in terms of linear combinations of $x_{10}, \ldots, x_{i-1,0}$. This immediately yields the following result.

LEMMA 2.2. Let $\mu$ be an eigenvalue of $T$ and let $\phi_{1}, \ldots, \phi_{s}$ be a canonical system of root polynomials having orders $\kappa_{1} \geq \cdots \geq \kappa_{s}$. Given any other root polynomial $\tilde{\phi}$ having order $\tilde{\kappa}$, it follows that $\tilde{\phi}(\mu)$ is a linear combination of all $\phi_{i}(\mu)$ satisfying $\kappa_{i} \geq \tilde{\kappa}$.

Proof. Let $i$ be the smallest integer such that $\kappa_{i}<\tilde{\kappa}$. Let us suppose that the statement of the lemma does not hold. Then there is a root polynomial $\tilde{\phi}$ 
of order $\tilde{\kappa}$ such that $\tilde{\phi}(\mu)$ cannot be expressed in terms of a linear combination of $\phi_{1}(\mu), \ldots, \phi_{i-1}(\mu)$. This, however, violates the maximality of $\phi_{i}(\mu)$ mentioned above.

The following lemma provides the connection between root polynomials and the nullspace of $\mathbb{T}_{\mu, r}$.

Lemma 2.3. Let $\mu \in \Omega, q \in\{0, \ldots, r-1\}$, and

$$
X=[\underbrace{0, \ldots, 0}_{q \text { times }}, x_{0}, \ldots, x_{r-q-1}] \in \mathbb{C}^{n \times r}
$$

with $x_{0} \neq 0$. Furthermore, let

$$
\phi_{X}(z):=\sum_{k=0}^{r-q-1} x_{k}(z-\mu)^{k} .
$$

Then

$$
\mathbb{T}_{\mu, r}(X)=[\underbrace{0, \ldots, 0}_{q \text { times }}, y_{0}, \ldots, y_{r-q-1}] \text {, where } y_{k}=\frac{1}{k !}\left(T \phi_{X}\right)^{(k)}(\mu) .
$$

Thus, $X \in \operatorname{ker} \mathbb{T}_{\mu, r}$ if and only if $\phi_{X}$ is a root polynomial of order at least $r-q$ for the eigenvalue $\mu$ of $T$.

Proof. Let $e_{j}$ denote the $j$ th unit vector of length $r$. Then for $0 \leq k \leq r-q-1$ it holds that

$$
\begin{aligned}
\mathbb{T}_{\mu, r}(X) e_{q+1+k} & =\frac{1}{2 \pi \mathrm{i}} \int_{\mathcal{C}} T(z) X\left((z-\mu) I_{r}-N_{r}\right)^{-1} e_{q+1+k} \mathrm{~d} z \\
& =\frac{1}{2 \pi \mathrm{i}} \int_{\mathcal{C}} T(z) X \sum_{i=0}^{q+k} \frac{1}{(z-\mu)^{i+1}} e_{q+1+k-i} \mathrm{~d} z \\
& =\frac{1}{2 \pi \mathrm{i}} \sum_{i=0}^{k} \int_{\mathcal{C}} \frac{1}{(z-\mu)^{i+1}} T(z) x_{k-i} \mathrm{~d} z \\
& =\sum_{i=0}^{k} \frac{1}{i !} T^{(i)}(\mu) x_{k-i}=\sum_{i=0}^{k} \frac{1}{i !(k-i) !} T^{(i)}(\mu) \phi_{X}^{(k-i)}(\mu) \\
& =\frac{1}{k !}\left(T \phi_{X}\right)^{(k)}(\mu) .
\end{aligned}
$$

Analogously one shows $\mathbb{T}_{\mu, r}(X) e_{j}=0$ for $j \leq q$. This completes the proof of (2.8).

As an immediate consequence of Lemma 2.3, we recover the fact that ker $\mathbb{T}_{\mu, r}=$ $\{0\}$ if and only if $\mu \in \Omega$ is not an eigenvalue of $T$.

For our further developments, observe that the multiplication of $N_{r}$ with $X \in$ $\mathbb{C}^{n \times r}$ affects a right shift of the columns of $X$, i.e., $X N_{r}=\mathrm{S}(X)$ with

$$
\mathrm{S}\left(\left[x_{0}, x_{1}, \ldots x_{r-1}\right]\right):=\left[0, x_{0}, \ldots, x_{r-2}\right] .
$$

From the relation $N_{r} J_{\mu, r}=J_{\mu, r} N_{r}$, it follows that $\mathbb{T}_{\mu, r}(\mathrm{~S}(X))=\mathrm{S}\left(\mathbb{T}_{\mu, r}(X)\right)$. Hence, the operator $\mathbf{S}$ commutes with $\mathbb{T}_{\mu, r}$. This yields that $\operatorname{ker} \mathbb{T}_{\mu, r}$ is $\mathrm{S}$-invariant:

$$
X \in \operatorname{ker} \mathbb{T}_{\mu, r} \Rightarrow \mathrm{S}(X) \in \operatorname{ker} \mathbb{T}_{\mu, r} .
$$

Copyright $@$ by SIAM. Unauthorized reproduction of this article is prohibited. 
The following theorem states that a basis of the nullspace can be constructed from a canonical system of root polynomials. An alternative derivation of this result can be found in [14, sect. 3.4].

TheOrem 2.4. Suppose that $\mu$ is an eigenvalue of $T$ with partial multiplicities $\kappa_{1} \geq \cdots \geq \kappa_{s} \geq 1$. Let $\phi_{1}, \ldots, \phi_{s}$ be a canonical system of root polynomials, as in (2.6), with coefficients $x_{i k} \in \mathbb{C}^{n}$. For $i=1, \ldots, s$ let

$$
X_{i}= \begin{cases}{\left[x_{i 0}, \ldots, x_{i, r-1}\right]} & \text { if } r \leq \kappa_{i} \\ {[\underbrace{0, \ldots, 0}_{r-\kappa_{i} \text { zeros }}, x_{i 0}, \ldots, x_{i, \kappa_{i}-1}]} & \text { if } r>\kappa_{i} .\end{cases}
$$

Then the matrices

$$
\mathrm{S}^{j}\left(X_{i}\right), \quad i=1, \ldots, s, \quad j=0, \ldots, \min \left\{\kappa_{i}, r\right\}-1,
$$

form a basis of $\operatorname{ker} \mathbb{T}_{\mu, r}$. In particular,

$$
\operatorname{dim} \operatorname{ker} \mathbb{T}_{\mu, r}=\sum_{i=1}^{s} \min \left\{\kappa_{i}, r\right\}
$$

Proof. By Lemma 2.3 and (2.9), all matrices defined in (2.10) are contained in $\operatorname{ker} \mathbb{T}_{\mu, r}$. Since the polynomials $\phi_{i}$ form a canonical system, the vectors $x_{i 0}, i=1, \ldots s$, are linearly independent. This immediately implies that the matrices $\mathrm{S}^{j}\left(X_{i}\right)$ defined in (2.10) form a linearly independent set.

It remains to show that any $X \in \operatorname{ker} \mathbb{T}_{\mu, r}$ can be expressed as a linear combination of the matrices $\mathrm{S}^{j}\left(X_{i}\right)$. Assuming that $X \neq 0$, let us partition

$$
X=[\underbrace{0, \ldots, 0}_{q \text { times }}, x_{0}, \ldots, x_{r-q-1}] \in \mathbb{C}^{n \times r}
$$

with $x_{0} \neq 0$. Then, according to Lemma 2.3, the vector polynomial $\phi_{X}$ defined in (2.7) is a root polynomial of order at least $r-q$. By Lemma 2.2, this implies that $x_{0}$ can be expressed as a linear combination

$$
x_{0}=\sum_{i=1}^{t} \alpha_{i} x_{i 0}
$$

where $t$ is the largest integer such that $\kappa_{t} \geq r-q$. Based on these scalars $\alpha_{i}$, let us now define

$$
\tilde{X}:=X-\sum_{i=1}^{t} \alpha_{i} \mathrm{~S}^{q_{i}}\left(X_{i}\right), \quad \text { with } \quad q_{i}= \begin{cases}q & \text { if } r \leq \kappa_{i}, \\ q-\left(r-\kappa_{i}\right) & \text { otherwise }\end{cases}
$$

Then (2.11) implies that the $(q+1)$ th column of $\tilde{X}$ becomes zero:

$$
\tilde{X}=[\underbrace{0, \ldots, 0}_{q+1 \text { times }}, \tilde{x}_{0}, \ldots, \tilde{x}_{r-q-2}] .
$$

We can repeat this process to successively annihilate columns $q+2, q+3, \ldots, r$. Eventually, this shows that $X$ is a linear combination of the matrices $\mathrm{S}^{j}\left(X_{i}\right)$. 
2.3. A rank characterization for $\sum_{j=1}^{s} m_{j}(T) \geq r$. According to the discussion in section 2.1, Theorem 2.4 implies the following characterization of the nullspace dimension of $\mathbb{T}_{C}$.

COROLlARY 2.5. Let $T: \Omega \rightarrow \mathbb{C}^{n \times n}$ be a regular analytic matrix-valued function and let $C \in \mathbb{C}^{r \times r}$ have the Jordan decomposition $C=S \operatorname{diag}\left(J_{\mu_{1}, r_{1}}, \ldots, J_{\mu_{q}, r_{q}}\right) S^{-1}$. Suppose that all eigenvalues $\mu_{j}$ of $C$ are contained in $\Omega$ and hence the linear operator $\mathbb{T}_{C}$ in (2.4) is well defined. Let $\mathcal{J}$ be the set of $j$ such that $\mu_{j}$ is an eigenvalue of $T$. For $j \in \mathcal{J}$ let $\kappa_{1 j} \geq \cdots \geq \kappa_{s_{j}, j} \geq 1$ be the partial multiplicities of $\mu_{j}$ as an eigenvalue of $T$. Then

$$
\operatorname{dim} \operatorname{ker} \mathbb{T}_{C}=\sum_{j \in \mathcal{J}} \sum_{i=1}^{s_{j}} \min \left\{\kappa_{i j}, r_{j}\right\} .
$$

In what follows, we will choose $C$ to be an upper triangular matrix of the form

$$
C(\mu, \Gamma):=\left[\begin{array}{cccc}
\mu_{1} & \gamma_{12} & \cdots & \gamma_{1 r} \\
0 & \mu_{2} & \ddots & \vdots \\
\vdots & \ddots & \ddots & \gamma_{r-1, r} \\
0 & \cdots & 0 & \mu_{r}
\end{array}\right],
$$

where $\mu:=\left[\begin{array}{lll}\mu_{1} & \ldots & \mu_{r}\end{array}\right] \in \mathbb{C}^{r}, \Gamma:=\left[\begin{array}{lll}\gamma_{12} & \ldots & \gamma_{r-1, r}\end{array}\right]^{T} \in \mathbb{C}^{r(r-1) / 2}$. We let the set $\mathcal{G}(\mu)$ contain all values of $\Gamma$ for which each eigenvalue of $C(\mu, \Gamma)$ has geometric multiplicity one. The set $\mathcal{G}(\mu)$ is dense in $\mathbb{C}^{r(r-1) / 2}$; see [12].

After these preparations, we are ready to present our main result, a dimensionality characterization for the eigenvalue multiplicities of $T$. Below, $\mathbb{S}^{r}$ denotes the set of $r$-tuples with elements in $\mathbb{S}$.

TheOrem 2.6. Let $T: \Omega \rightarrow \mathbb{C}^{n \times n}$ be an analytic matrix-valued function. Then the following two conditions are equivalent:

(i) $\sum_{j=1}^{s} m_{j}(T) \geq r$.

(ii) There exists $\mu \in \mathbb{S}^{r}$ such that

$$
\operatorname{dim}\left\{X \mid \mathbb{T}_{C(\mu, \Gamma)}(X)=0\right\} \geq r
$$

for all $\Gamma \in \mathcal{G}(\mu)$.

Proof. The result follows directly from Corollary 2.5 , by letting $\mu$ contain at most $m_{j}(T)$ copies of each eigenvalue $\lambda_{j}$.

Using basic properties of the Kronecker product, the $n r \times n r$ matrix representation $\mathcal{K}(\mu, \Gamma)$ of the linear operator $\mathbb{T}_{C(\mu, \Gamma)}$ can be easily derived from $(2.4)$ :

$$
\mathcal{K}(\mu, \Gamma, T)=\frac{1}{2 \pi \mathrm{i}} \int_{\mathcal{C}}(z I-C(\mu, \Gamma))^{-T} \otimes T(z) \mathrm{d} z .
$$

Equivalently, the decomposition (2.2) of $T$ and the definition (2.3) of $\mathbb{T}_{C}$ give

$$
\mathcal{K}(\mu, \Gamma, T)=\sum_{k=1}^{K} f_{k}(C(\mu, \Gamma))^{T} \otimes A_{k} .
$$

Using this matrix representation, (2.13) becomes equivalent to the rank condition

$$
\operatorname{rank} \mathcal{K}(\mu, \Gamma, T) \leq n \cdot r-r .
$$

Copyright (c) by SIAM. Unauthorized reproduction of this article is prohibited. 
3. Derivation of the singular value characterization. Coming back to our proposed distance $\tau_{r}(\mathbb{S})$ defined in (1.4), the rank characterization (2.15) derived in the previous section yields

$$
\begin{aligned}
\tau_{r}(\mathbb{S}) & =\inf \left\{\|\Delta\|_{2} \mid \exists \mu \in \mathbb{S}^{r} \text { such that } \operatorname{rank}(\mathcal{K}(\mu, \Gamma, T+\Delta)) \leq n \cdot r-r \text { for } \Gamma \in \mathcal{G}(\mu)\right\} \\
& =\inf _{\mu \in \mathbb{S}^{r}} \mathcal{P}_{r}(\mu),
\end{aligned}
$$

where $\tilde{T}:=T+\Delta$ denotes the analytic matrix-valued function $\tilde{T}(\lambda)=T(\lambda)+\Delta$, and

$$
\mathcal{P}_{r}(\mu):=\inf \left\{\|\Delta\|_{2} \mid \operatorname{rank}(\mathcal{K}(\mu, \Gamma, T+\Delta)) \leq n \cdot r-r\right\}
$$

for any $\Gamma \in \mathcal{G}(\mu)$. This section is concerned with the derivation of a singular value optimization characterization for $\mathcal{P}_{r}(\mu)$.

We immediately obtain the lower bound

$$
\mathcal{P}_{r}(\mu) \geq \sigma_{-r}(\mathcal{K}(\mu, \Gamma, T))
$$

from the Eckart-Young theorem, where $\sigma_{-r}(\cdot)$ denotes the $r$ th smallest singular value of a matrix. Indeed, since the above inequality holds for any $\Gamma \in \mathcal{G}(\mu)$, and due to the continuity of the singular value $\sigma_{-r}(\cdot)$ with respect to $\Gamma$, we deduce

$$
\mathcal{P}_{r}(\mu) \geq \sup _{\Gamma} \sigma_{-r}(\mathcal{K}(\mu, \Gamma, T))=: \kappa_{r}(\mu)
$$

As in the derivations for the specialized cases [22, 19], which led us to consider this more general setting, we prove $\mathcal{P}_{r}(\mu)=\kappa_{r}(\mu)$, under mild multiplicity and linear independence assumptions, by constructing a perturbation $\Delta_{*}$ such that

(i) $\left\|\Delta_{*}\right\|_{2}=\kappa_{r}(\mu)$ and

(ii) $\operatorname{rank}\left(\mathcal{K}\left(\mu, \Gamma, T+\Delta_{*}\right)\right) \leq n \cdot r-r$ for some $\Gamma \in \mathcal{G}(\mu)$.

As discussed in [19], the supremum in (3.2) is always attained at some $\Gamma_{*}$, as long as $r \leq n$ and for generic values of $\mu$. More precisely, we require the vector $\mu \in \mathbb{C}^{r}$ to satisfy the condition that the matrix

$$
T\left[\mu_{k}, \mu_{l}\right]:= \begin{cases}\left(T\left(\mu_{l}\right)-T\left(\mu_{k}\right)\right) /\left(\mu_{l}-\mu_{k}\right), & \mu_{l} \neq \mu_{k}, \\ T^{\prime}\left(\mu_{l}\right), & \mu_{l}=\mu_{k},\end{cases}
$$

is invertible for all $k \neq l$. The proof for the existence of $\Gamma_{*}$ can be found in [19, Appendix A]. Although this proof targets matrix polynomials $P(\lambda)$, it makes no use of particular properties of polynomials and directly carries over to an analytic matrix-valued function $T(\lambda)$ represented in the form (2.2).

Let $\Gamma_{*}$ be such that

$$
\kappa_{r}(\mu)=\sigma_{-r}\left(\mathcal{K}\left(\mu, \Gamma_{*}, T\right)\right)
$$

and let $U, V \in \mathbb{C}^{n r}$ be the associated left and right singular vectors satisfying

$$
\mathcal{K}\left(\mu, \Gamma_{*}, T\right) V=\kappa_{r}(\mu) U \quad \text { and } \quad U^{*} \mathcal{K}\left(\mu, \Gamma_{*}, T\right)=\kappa_{r}(\mu) V^{*} .
$$

Let us consider the perturbation defined by

$$
\Delta_{*}:=-\kappa_{r}(\mu) \mathcal{U} \mathcal{V}^{+},
$$

Copyright $@$ by SIAM. Unauthorized reproduction of this article is prohibited. 
where $\mathcal{U}, \mathcal{V} \in \mathbb{C}^{n \times r}$ are such that $\operatorname{vec}(\mathcal{U})=U$, $\operatorname{vec}(\mathcal{V})=V$, and $\mathcal{V}^{+}$denotes the Moore-Penrose pseudoinverse of $\mathcal{V}$. We claim that $\Delta_{*}$ satisfies both properties (i) and (ii) under the following mild assumptions.

DEFINITION 3.1 (multiplicity assumption). The multiplicity of the singular value $\sigma_{-r}\left(\mathcal{K}\left(\mu, \Gamma_{*}, T\right)\right)$ is one.

DEFINITION 3.2 (linear independence assumption). There exists a right singular vector $V$ associated with the singular value $\sigma_{-r}\left(\mathcal{K}\left(\mu, \Gamma_{*}, T\right)\right)$ such that the $n \times r$ matrix $\mathcal{V}$ with $\operatorname{vec}(\mathcal{V})=V$ has full column rank.

The subsequent two subsections are devoted to the proofs of properties (i) and (ii) for the perturbation $\Delta_{*}$ defined by (3.4). To simplify the derivation, we make two additional assumptions initially. First, $\mu$ is supposed to be comprised of distinct elements. In this case all eigenvalues of $C(\mu, \Gamma)$ are simple, and hence $\mathcal{G}(\mu)=\mathbb{C}^{r(r-1) / 2}$. Second, we assume that $T\left[\mu_{k}, \mu_{l}\right]$ has full rank for all $k \neq l$. Eventually, these two additional assumptions will be dropped.

3.1. Property (i): Norm of $\boldsymbol{\Delta}_{*}$. In this section, we show $\left\|\Delta_{*}\right\|_{2}=\kappa_{r}(\mu)=$ $\sigma_{-r}\left(\mathcal{K}\left(\mu, \Gamma_{*}, T\right)\right)$, which amounts to verifying $\left\|\mathcal{U} \mathcal{V}^{+}\right\|_{2}=1$. As noted in $[24,22,19]$, the latter property follows from the relation $\mathcal{U}^{*} \mathcal{U}=\mathcal{V}^{*} \mathcal{V}$, which we will establish under the multiplicity assumption.

Let us consider the function

$\sigma(\Gamma):=\sigma_{-r}(\mathcal{K}(\mu, \Gamma, T)), \quad$ where $\quad \mathcal{K}(\mu, \Gamma, T):=\frac{1}{2 \pi \mathrm{i}} \int_{\mathcal{C}}(z I-C(\mu, \Gamma))^{-T} \otimes T(z) \mathrm{d} z$

By differentiating the relation $I=(z I-C(\mu, \Gamma)) R(z, \Gamma)$ one obtains the following identities for the partial derivatives of the resolvent $R(z, \Gamma)=(z I-C(\mu, \Gamma))^{-1}$ with respect to the real and imaginary parts of the components $\gamma_{i k}$ of $\gamma$ :

$$
\begin{aligned}
& \frac{\partial R}{\partial \Re \gamma_{i k}}(z, \Gamma)=-R(z, \Gamma) \frac{\partial C}{\partial \Re \gamma_{i k}}(z, \Gamma) R(z, \Gamma)=-R(z, \Gamma) e_{i} e_{k}^{T} R(z, \Gamma), \\
& \frac{\partial R}{\partial \Im \gamma_{i k}}(z, \Gamma)=-R(z, \Gamma) \frac{\partial C}{\partial \Im \gamma_{i k}}(z, \Gamma) R(z, \Gamma)=-\mathrm{i} R(z, \Gamma) e_{i} e_{k}^{T} R(z, \Gamma),
\end{aligned}
$$

where $1 \leq i<k \leq r$. It follows that

$$
\begin{aligned}
& \frac{\partial \mathcal{K}}{\partial \Re \gamma_{i k}}(\mu, \Gamma, T)=-\frac{1}{2 \pi \mathrm{i}} \int_{\mathcal{C}}\left[R(z, \Gamma) e_{i} e_{k}^{T} R(z, \Gamma)\right]^{T} \otimes T(z) \mathrm{d} z, \\
& \frac{\partial \mathcal{K}}{\partial \Im \gamma_{i k}}(\mu, \Gamma, T)=-\frac{1}{2 \pi} \int_{\mathcal{C}}\left[R(z, \Gamma) e_{i} e_{k}^{T} R(z, \Gamma)\right]^{T} \otimes T(z) \mathrm{d} z
\end{aligned}
$$

for $1 \leq i<k \leq r$.

Let

$$
G:=\frac{1}{2 \pi \mathrm{i}} \int_{\mathcal{C}} R\left(z, \Gamma_{*}\right) \mathcal{U}^{*} T(z) \mathcal{V} R\left(z, \gamma_{*}\right) \mathrm{d} z
$$

From the assumption that the singular value $\sigma\left(\Gamma_{*}\right)$ is simple, it follows that the 
function $\gamma \mapsto \sigma(\Gamma)$ is analytic $[10]$ at $\Gamma_{*}$, and

$$
\begin{aligned}
0 & =\frac{\partial \sigma}{\partial \Re \gamma_{i k}}\left(\Gamma_{*}\right)=\Re\left(U^{*} \frac{\partial \mathcal{K}}{\partial \Re \gamma_{i k}}\left(\mu, \Gamma_{*}, T\right) V\right)=\Re\left(\operatorname{vec}(\mathcal{U})^{*} \frac{\partial \mathcal{K}}{\partial \Re \gamma_{i k}}\left(\mu, \Gamma_{*}, T\right) \operatorname{vec}(\mathcal{V})\right) \\
& =-\Re\left(\operatorname{vec}(\mathcal{U})^{*} \operatorname{vec}\left(\frac{1}{2 \pi \mathrm{i}} \int_{\mathcal{C}} T(z) \mathcal{V} R\left(z, \gamma_{*}\right) e_{i} e_{k}^{T} R\left(z, \Gamma_{*}\right) \mathrm{d} z\right)\right) \\
& =-\Re\left(\operatorname{tr}\left(\frac{1}{2 \pi \mathrm{i}} \int_{\mathcal{C}} \mathcal{U}^{*} T(z) \mathcal{V} R\left(z, \Gamma_{*}\right) e_{i} e_{k}^{T} R\left(z, \Gamma_{*}\right) \mathrm{d} z\right)\right) \\
& =-\Re\left(e_{k}^{T} G e_{i}\right) \quad \text { for } 1 \leq i<k \leq r,
\end{aligned}
$$

where the last equality follows from the trace identity $\operatorname{tr}(X Y)=\operatorname{tr}(Y X)$. Analogously we have

$$
0=\frac{\partial \sigma}{\partial \Im \gamma_{i k}}\left(\Gamma_{*}\right)=\Re\left(-\mathrm{i} e_{k}^{T} G e_{i}\right)=\Im\left(e_{k}^{T} G e_{i}\right) \quad \text { for } 1 \leq k<i \leq r .
$$

Thus, $G$ is upper triangular. The identities

$$
\begin{gathered}
\frac{1}{2 \pi \mathrm{i}} \int_{\mathcal{C}} T(z) \mathcal{V}(z I-C(\mu, \Gamma))^{-1} \mathrm{~d} z=\sigma\left(\Gamma_{*}\right) \mathcal{U}, \\
\frac{1}{2 \pi \mathrm{i}} \int_{\mathcal{C}}(z I-C(\mu, \Gamma))^{-1} \mathcal{U}^{*} T(z) \mathrm{d} z=\sigma\left(\Gamma_{*}\right) \mathcal{V}^{*}
\end{gathered}
$$

imply

$$
\begin{aligned}
& \left(z I-C\left(\mu, \Gamma_{*}\right)\right) G=\frac{1}{2 \pi \mathrm{i}} \int_{\mathcal{C}} \mathcal{U}^{*} T(z) \mathcal{V}(z I-C(\mu, \Gamma))^{-1} \mathrm{~d} z=\sigma\left(\Gamma_{*}\right) \mathcal{U}^{*} \mathcal{U} \\
& G\left(z I-C\left(\mu, \Gamma_{*}\right)\right)=\frac{1}{2 \pi \mathrm{i}} \int_{\mathcal{C}}(z I-C(\mu, \Gamma))^{-1} \mathcal{U}^{*} T(z) \mathcal{V} \mathrm{d} z=\sigma\left(\Gamma_{*}\right) \mathcal{V}^{*} \mathcal{V}
\end{aligned}
$$

Thus,

$$
\sigma\left(\Gamma_{*}\right)\left(\mathcal{U}^{*} \mathcal{U}-\mathcal{V}^{*} \mathcal{V}\right)=G C\left(\mu, \Gamma_{*}\right)-C\left(\mu, \Gamma_{*}\right) G .
$$

Consequently, since both $G$ and $C\left(\mu, \Gamma_{*}\right)$ are upper triangular, the right-hand side of the equation above is strictly upper triangular, whereas the left-hand side is Hermitian. Therefore, both sides must vanish, and we have $\mathcal{U}^{*} \mathcal{U}=\mathcal{V}^{*} \mathcal{V}$ as desired.

3.2. Property (ii): Rank condition. It remains to show that the constructed perturbation $\Delta_{*}=-\kappa_{r}(\mu) \mathcal{U} \mathcal{V}^{+}$satisfies the rank property (ii) for $\mathcal{K}$. Using both the multiplicity and linear independence assumptions, we will establish this property by showing that the nullspace of the perturbed linear operator has dimension at least $r$.

We start by writing the left-hand equation in (3.3) in terms of the operator $\mathbb{T}_{C\left(\mu, \gamma_{*}\right)}$ :

$$
\mathbb{T}_{C\left(\mu, \gamma_{*}\right)}(\mathcal{V})=\sigma\left(\Gamma_{*}\right) \mathcal{U}
$$

By exploiting the property $\mathcal{U}^{*} \mathcal{U}=\mathcal{V}^{*} \mathcal{V}$ from the previous section, we have $\mathcal{U}=\mathcal{U} \mathcal{V}^{+} \mathcal{V}$ and hence

$$
0=\mathbb{T}_{C\left(\mu, \Gamma_{*}\right)}(\mathcal{V})-\sigma\left(\gamma_{*}\right) \mathcal{U} \mathcal{V}^{+} \mathcal{V}=\mathbb{T}_{C\left(\mu, \Gamma_{*}\right)}(\mathcal{V})+\Delta_{*} \mathcal{V}
$$

Copyright (c) by SIAM. Unauthorized reproduction of this article is prohibited. 
Furthermore, consider the space of matrices

$$
\left\{D \mid C\left(\mu, \Gamma_{*}\right) D-D C\left(\mu, \Gamma_{*}\right)=0\right\}
$$

commuting with $C\left(\mu, \Gamma_{*}\right)$, which has dimension at least $r$ [15, Theorem $1, \mathrm{p}$. 219]. It is easy to see that any such $D$ also commutes with $\left(z I-C\left(\mu, \Gamma_{*}\right)\right)^{-1}$, and hence

$$
\begin{aligned}
0 & =\mathbb{T}_{C\left(\mu, \Gamma_{*}\right)}(V) D+\Delta_{*} V D=\frac{1}{2 \pi \mathrm{i}} \int_{\mathcal{C}} T(z) V D(z I-C(\mu, \Gamma))^{-1} \mathrm{~d} z+\Delta_{*} V D \\
& =\mathbb{T}_{C\left(\mu, \Gamma_{*}\right)}(V D)+\Delta_{*} V D .
\end{aligned}
$$

This shows the desired result, that is, the nullspace of $X \mapsto \mathbb{T}_{C\left(\mu, \Gamma_{*}\right)}(X)+\Delta_{*} X$ has dimension at least $r$.

4. Main result. Before stating the main result, we discuss why the two additional assumptions mentioned above can be removed. Suppose that $\mu \in \mathbb{C}^{r}$ has repeating elements and/or that $T\left[\mu_{k}, \mu_{l}\right]$ is singular for some $k, l$, but let us still assume that the linear independence and multiplicity assumptions hold at $\mu$. By the regularity of $T$, it follows that essentially for all $\tilde{\mu} \in \mathbb{C}^{r}$ with mutually distinct elements and sufficiently close to $\mu$, we have that $T\left[\tilde{\mu}_{l}, \tilde{\mu}_{k}\right]$ is nonsingular for all $k, l$. By continuity of the singular value and vectors, it follows that the multiplicity and linear independence assumptions remain valid for $\tilde{\mu}$, provided that $\tilde{\mu}$ is chosen sufficiently close to $\mu$. Consequently, it follows from the previous section that $\kappa_{r}(\tilde{\mu})=\mathcal{P}_{r}(\tilde{\mu})$ for all such $\tilde{\mu}$. By continuity, $\kappa_{r}(\mu)=\mathcal{P}_{r}(\mu)$.

The following theorem summarizes our findings.

TheOREM 4.1. Let $T: \Omega \rightarrow \mathbb{C}^{n \times n}$ be analytic and regular. Suppose that $\mathbb{S}=$ $\left\{\lambda_{1}, \ldots, \lambda_{s}\right\} \subset \Omega$ and $r \leq n$ are given. Then the distance $\tau_{r}(\mathbb{S})$ defined in (1.4) has the singular value optimization characterization

$$
\tau_{r}(\mathbb{S})=\inf _{\mu \in \mathbb{S}^{r}} \sup _{\Gamma \in \mathbb{C}^{r(r-1) / 2}} \sigma_{-r}(\mathcal{K}(\mu, \Gamma, T)),
$$

provided that the multiplicity and linear independence assumptions hold at $\mu_{*}$, the optimal value of $\mu$. Moreover, a minimal perturbation with 2-norm equal to $\tau_{r}(\mathbb{S})$ is given by (3.4).

Let us remark that the statement of Theorem 4.1 remains true for $r>n$, under the additional assumption that the inner supremum in (4.1) is attained.

5. Divided-difference formulas. The singular value characterization (4.1) can be conveniently expressed in terms of divided differences; see, e.g., [9]. The divided difference of a function $f: \mathbb{R} \rightarrow \mathbb{R}$ at nodes $x_{0}, \ldots, x_{k}$ is given by

$$
f\left[x_{0}, x_{1}, \ldots, x_{k}\right]= \begin{cases}\frac{f\left[x_{1}, \ldots, x_{k}\right]-f\left[x_{0}, \ldots, x_{k-1}\right]}{x_{k}-x_{0}}, & x_{0} \neq x_{k}, \\ \frac{f^{(k)}\left(x_{0}\right)}{k !}, & x_{0}=x_{k},\end{cases}
$$

where the nodes are assumed to be contagious; that is, $x_{j}=x_{\ell}$ for some $\ell>j$ implies $x_{i}=x_{j}$ for $i=j, \ldots, \ell$. Given a lower triangular matrix $L$, the entries of the matrix function $F=f(L)$, which is also lower triangular, are given by

$$
f_{i \ell}=\sum_{\left(s_{0}, s_{1}, \ldots, s_{k}\right)} l_{s_{1} s_{0}} l_{s_{2} s_{1}} \ldots l_{s_{k} s_{k-1}} f\left[\mu_{s_{0}}, \ldots, \mu_{s_{k}}\right]
$$

for $i>\ell$ and $f_{i i}=f\left(\mu_{i}\right)$, where $\mu_{i}=l_{i i}$ and the summation is over all increasing sequences of integers starting with $\ell$ and ending with $i$; see [18, Theorem 4.11]. 
Applying the formula above for functions of lower triangular matrices to

$$
\mathcal{K}(\mu, \Gamma, T)=\sum_{k=1}^{K} f_{k}(C(\mu, \gamma))^{T} \otimes A_{k}
$$

yields the following result: The matrix $\mathcal{K}(\mu, \Gamma, T) \in \mathbb{C}^{n r \times n r}$ is block lower triangular and its $n \times n$ submatrix at rows $1+(i-1) n: i n$ and at columns $1+(\ell-1) n: \ell n$ is given by

$$
\begin{cases}\sum_{\left(s_{0}, s_{1}, \ldots, s_{k}\right)} \gamma_{s_{1} s_{0}} \gamma_{s_{2} s_{1}} \ldots \gamma_{s_{k} s_{k-1}} T\left[\mu_{s_{0}}, \ldots, \mu_{s_{k}}\right], & i>\ell \\ T\left(\mu_{i}\right), & i=\ell \\ 0, & i<\ell\end{cases}
$$

The proof of these relations for matrix polynomials can be found in [19, sect. 3.4]; it directly extends to analytic matrix-valued functions.

When $\mathbb{S}=\{\lambda\}$ and $r=2$, Theorem 4.1 combined with the representation of $\mathcal{K}(\mu, \Gamma, T)$ above results in the formula

$$
\sup _{\gamma \in \mathbb{C}} \sigma_{-2}\left(\left[\begin{array}{cc}
T(\lambda) & 0 \\
\gamma \cdot T^{\prime}(\lambda) & T(\lambda)
\end{array}\right]\right)
$$

for the distance to a nearest nonlinear eigenvalue problem with $\lambda$ as a multiple eigenvalue. When two eigenvalues are prescribed (that is, $\mathbb{S}=\left\{\lambda_{1}, \lambda_{2}\right\}$ and $r=2$ ), we obtain

$$
\inf _{\mu_{1}, \mu_{2} \in \mathbb{S}^{2}} \sup _{\gamma \in \mathbb{C}} \sigma_{-2}\left(\left[\begin{array}{cc}
T\left(\mu_{1}\right) & 0 \\
\gamma \cdot T\left[\mu_{1}, \mu_{2}\right] & T\left(\mu_{2}\right)
\end{array}\right]\right) .
$$

6. Numerical examples. We illustrate the main result on two examples. The first is a small-scale problem, concerning the distance to a nearest nonlinear eigenvalue problem with a multiple eigenvalue. The second one is a large-scale problem, and is aimed at illustrating the point that even if the individual backward errors are small for two approximate eigenvalues, there may not be any nearby problem with both of these two eigenvalues. Furthermore, it is not possible to conclude this by solely looking at the resolvent norms.

6.1. Prescribing a multiple eigenvalue. Let us consider the distance $\tau_{r}(\mathbb{S})$ with $\mathbb{S}=\{\lambda\}$ and $r=2$ for a prescribed eigenvalue $\lambda \in \mathbb{C}$. In this case, the formula (5.2) applies. In fact, the supremum can be taken over $\mathbb{R}$ instead of $\mathbb{C}$, since the singular values of

$$
\left[\begin{array}{cc}
T(\lambda) & 0 \\
\gamma \cdot T^{\prime}(\lambda) & T(\lambda)
\end{array}\right] \text { and }\left[\begin{array}{cc}
T(\lambda) & 0 \\
|\gamma| \cdot T^{\prime}(\lambda) & T(\lambda)
\end{array}\right]
$$

are identical. We deduce that the distance to a nearest nonlinear eigenvalue problem with a multiple eigenvalue can be expressed as

$$
\inf _{\lambda \in \Omega} \sup _{\gamma \in \mathbb{R}} \sigma_{-2}\left(\left[\begin{array}{cc}
T(\lambda) & 0 \\
\gamma \cdot T^{\prime}(\lambda) & T(\lambda)
\end{array}\right]\right) \text {. }
$$

In view of the result by Malyshev [24] for the linear case, we conjecture that (6.1) holds without requiring the multiplicity and linear independence assumptions. 


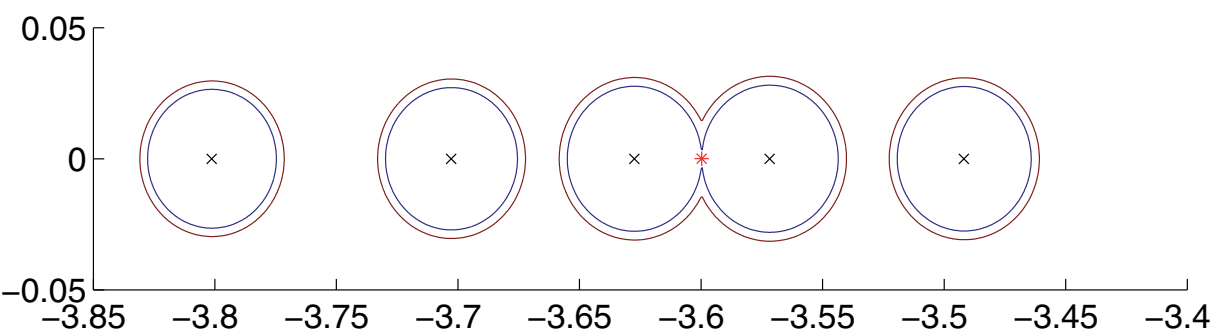

FIG. 1. Contours of the pseudospectra for the nonlinear eigenvalue problem (6.2), with the eigenvalues marked by crosses. The inner contour corresponds to the $\epsilon$-pseudospectrum for $\epsilon$ equal to the distance to a nearest nonlinear eigenvalue problem with a multiple eigenvalue. Two components coalesce as expected, and the point of coalescence marked with an asterisk is the multiple eigenvalue of a nearest nonlinear eigenvalue problem.

It is well known for the standard eigenvalue problem $T(\lambda)=A-\lambda I$ that the distance to a nearest matrix with a multiple eigenvalue corresponds to the smallest $\epsilon$ such that two components of the $\epsilon$-pseudospectrum coalesce [3]. Generalizations of this result to matrix pencils and matrix polynomials, allowing for perturbations to all coefficients, have been shown in [2, Theorem 5.1] and [1, Theorem 7.1], respectively. The generalization to analytic matrix-valued functions in our setting, when only constant perturbations are permitted, appears to be straightforward. In particular, denoting the set of eigenvalues with $\Lambda(\cdot)$, we consider the following $\epsilon$-pseudospectrum for the analytic matrix-valued function $T$ :

$$
\Lambda_{\epsilon}(T):=\bigcup_{\|\Delta\|_{2} \leq \epsilon} \Lambda(T(\lambda)+\Delta)=\left\{\lambda \in \mathbb{C} \mid \sigma_{-1}(T(\lambda)) \leq \epsilon\right\} .
$$

The eigenvalues of $T$ are the only local minimizers of $\sigma_{-1}(T(\cdot))$ [11, Theorem 4.2], so there is a connected component of $\Lambda_{\epsilon}(T)$ around each eigenvalue of $T$. When $T$ has distinct eigenvalues, the smallest $\epsilon$ such that two components of $\Lambda_{\epsilon}(T)$ coalesce is the distance to a nearest nonlinear eigenvalue problem with a multiple eigenvalue, which admits the singular value characterization (6.1).

To illustrate (6.1), let us compute the distance to a multiple eigenvalue for a nonlinear eigenvalue problem from a delay differential equation [29]:

$$
T(\lambda)=\left(e^{\lambda}-1\right) B_{1}+\lambda^{2} B_{2}-B_{0} .
$$

For this purpose, we apply the algorithm in [26] to the characterization (6.1). The matrices $B_{0}, B_{1}, B_{2} \in \mathbb{R}^{8 \times 8}$ are given by

$$
\begin{aligned}
& B_{0}=100 \cdot I_{8}, \\
& B_{1}=\left[b_{j k}^{(1)}\right], \quad b_{j k}^{(1)}=[9-\max (j, k)] \cdot j \cdot k, \\
& B_{2}=\left[b_{j k}^{(2)}\right], \quad b_{j k}^{(2)}=9 \cdot \delta_{j k}+\frac{1}{j+k},
\end{aligned}
$$

with $\delta_{j k}=1$ if $j=k$, and zero otherwise. This is an example taken from [29]. A plot of the pseudospectra of $T$ is provided in Figure 1. The inner curves correspond to the boundary of $\Lambda_{\epsilon}(T)$ for $\epsilon=1.606$, which is the computed distance.

6.2. Checking that a pair of eigenvalue approximations corresponds to a nearby pair of eigenvalues. The second example is taken from [4], and concerns 


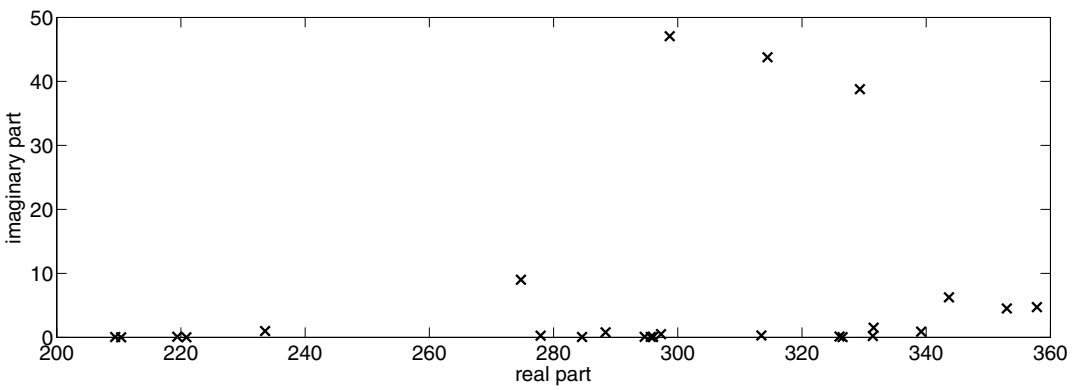

FIG. 2. Eigenvalues of nonlinear eigenvalue problem (6.3).

TABLE 1

Two pairs of eigenvalue approximations for the nonlinear eigenvalue problem (6.3): Individual backward errors (columns 3 and 4 ) and the distance (column 5) to a nearest problem having $z_{1}^{(j)}, z_{2}^{(j)}$ as exact eigenvalues.

\begin{tabular}{|l|l|l|l|l|l|}
\hline & $\mu_{1}^{(j)}$ & $\mu_{2}^{(j)}$ & $\sigma_{-1}\left(T\left(\mu_{1}^{(j)}\right)\right)$ & $\sigma_{-1}\left(T\left(\mu_{2}^{(j)}\right)\right)$ & $\tau_{2}\left(\left\{\mu_{1}^{(j)}, \mu_{2}^{(j)}\right\}\right)$ \\
\hline$j=1$ & $233.5+0.8 \mathrm{i}$ & $234+\mathrm{i}$ & 0.2863 & 0.6477 & 10.4347 \\
\hline$j=2$ & 326 & $326+0.5 \mathrm{i}$ & 0.1857 & 0.4993 & 0.6207 \\
\hline
\end{tabular}

the eigenvalues of

$$
T(\lambda)=K-\lambda^{2} \cdot M+i \cdot \lambda \cdot W_{1}+i \cdot \sqrt{\lambda^{2}-\sigma^{2}} \cdot W_{2},
$$

where $K, M, W_{1}, W_{2} \in \mathbb{R}^{9956 \times 9956}$ are symmetric, $K$ is positive semidefinite, $M$ is positive definite, and $\sigma=108.8774$. This nonlinear eigenvalue problem occurs from a model of the radio-frequency gun cavity [23]. Figure 2 displays some of the eigenvalues of $T$, computed with the algorithm described in [13].

Suppose now that a (different) algorithm returns two pairs of eigenvalue approximations:

$$
\mu_{1}^{(1)}=233.5+0.8 \mathrm{i}, \mu_{2}^{(1)}=234+\mathrm{i} \quad \text { and } \quad \mu_{1}^{(2)}=326, \mu_{2}^{(2)}=326+0.5 \mathrm{i} .
$$

Considered individually, each of the approximations gives a reasonably small backward error $\sigma_{-1}(T(\mu)$ ); see Table 1 . However, this does not imply that each pair is a good approximation to an eigenvalue pair of $T$. In fact, it can already be seen from Figure 2 that there is only one eigenvalue (with multiplicity 1 ) close to the first pair $\mu_{1}^{(1)}, \mu_{2}^{(1)}$. Hence, one of the two approximations is spurious. This can be verified by computing $\tau_{2}\left(\left\{\mu_{1}^{(1)}, \mu_{2}^{(1)}\right\}\right)$, which gives a much larger value than the individual backward errors; see column 5 of Table 1 . In contrast, the second pair $\mu_{1}^{(2)}, \mu_{2}^{(2)}$ is visually close to two eigenvalues. This is also confirmed by the fact that the distance $\tau_{2}\left(\left\{\mu_{1}^{(2)}, \mu_{2}^{(2)}\right\}\right)$ is of the same order as the individual backward errors.

Finally, Figure 3 illustrates

$$
\sigma_{-2}\left(\left[\begin{array}{cc}
T\left(\mu_{1}^{(2)}\right) & 0 \\
\gamma \cdot T\left[\mu_{1}^{(2)}, \mu_{2}^{(2)}\right] & T\left(\mu_{2}^{(2)}\right)
\end{array}\right]\right)
$$

with respect to $\gamma \in[0,3]$. The figure suggests that the singular value function is unimodal for a single parameter, which can be used in the optimization algorithm. 




FIG. 3. The plot of $\sigma_{-2}(\mathcal{K}(\mu, \gamma, T))$ with respect to $\gamma \in[0,3]$ for $\mu=\left[\mu_{1}^{(2)}, \mu_{2}^{(2)}\right]$, where $\mu_{1}^{(2)}, \mu_{2}^{(2)}$ are as in Table 1.

7. Concluding remarks. We have derived a further generalization of earlier works $[22,19]$ for locating nearest analytic matrix-valued functions with specified eigenvalues. For this purpose, we have studied the structure of the nullspace for Sylvester-like operators associated with nonlinear eigenvalue problems. We believe that these auxiliary results could be of independent interest. Our main result is the singular value characterization in Theorem 4.1. This characterization can be turned into a numerical method by exploiting the smoothness properties of singular values, using, for instance, the algorithm described in [26].

Acknowledgments. We thank Cedric Effenberger for inspiring discussions on the nullspace of $\mathbb{T}_{C}$ and for providing Figure 2. We are also grateful to two anonymous referees and Francoise Tisseur for their invaluable comments.

\section{REFERENCES}

[1] S. S. Ahmad and R. Alam, Pseudospectra, critical points and multiple eigenvalues of matrix polynomials, Linear Algebra Appl., 430 (2009), pp. 1171-1195.

[2] S. S. Ahmad, R. Alam, And R. Byers, On pseudospectra, critical points, and multiple eigenvalues of matrix pencils, SIAM J. Matrix Anal. Appl., 31 (2010), pp. 1915-1933.

[3] R. Alam And S. Bora, On sensitivity of eigenvalues and eigendecompositions of matrices, Linear Algebra Appl., 396 (2005), pp. 273-301.

[4] T. Betcke, N. J. Higham, V. Mehrmann, C. Schröder, and F. Tisseur, NLEVP: A collection of nonlinear eigenvalue problems, ACM Trans. Math. Software, 39 (2013), 7.

[5] T. Betcke and D. Kressner, Perturbation, extraction and refinement of invariant pairs for matrix polynomials, Linear Algebra Appl., 435 (2011), pp. 514-536.

[6] W.-J. BEYN, An integral method for solving nonlinear eigenvalue problems, Linear Algebra Appl., 436 (2012), pp. 3839-3863.

[7] W.-J. Beyn, C. Effenberger, And D. Kressner, Continuation of eigenvalues and invariant pairs for parameterized nonlinear eigenvalue problems, Numer. Math., 119 (2011), pp. 489516.

[8] D. Bindel And A. Hood, Localization theorems for nonlinear eigenvalue problems, SIAM J. Matrix Anal. Appl., 34 (2013), pp. 1728-1749.

[9] C. De Boor, Divided differences, Surv. Approx. Theory, 1 (2005), pp. 46-69.

[10] A. Bunse-Gerstner, R. Byers, V. Mehrmann, and N. K. Nichols, Numerical computation of an analytic singular value decomposition of a matrix valued function, Numer. Math., 
60 (1991), pp. 1-39.

[11] J. V. Burke, A. S. Lewis, And M. L. Overton, Optimization and pseudospectra, with applications to robust stability, SIAM J. Matrix Anal. Appl., 25 (2003), pp. 80-104.

[12] J. W. Demmel and A. Edelman, The dimension of matrices (matrix pencils) with given Jordan (Kronecker) canonical forms, Linear Algebra Appl., 230 (1995), pp. 61-87.

[13] C. Effenberger, Robust Successive Computation of Eigenpairs for Nonlinear Eigenvalue Problems, Technical report, MATHICSE, EPFL, Lausanne, Switzerland, 2012.

[14] C. Effenberger, Robust Solution Methods for Nonlinear Eigenvalue Problems, Ph.D. thesis, EPFL, Lausanne, Switzerland, 2013, available online at http://anchp.epfl.ch/students.

[15] F. R. Gantmacher, The Theory of Matrices, Vol. 1, Chelsea, New York, 1959.

[16] I. Gohberg, M. A. KaAshoek, And F. van Schagen, On the local theory of regular analytic matrix functions, Linear Algebra Appl., 182 (1993), pp. 9-25.

[17] I. Gohberg, P. Lancaster, and L. Rodman, Matrix Polynomials, Computer Science and Applied Mathematics, Academic Press [Harcourt Brace Jovanovich Publishers], New York, London, 1982.

[18] N. J. Higham, Functions of Matrices - Theory and Computation, SIAM, Philadelphia, 2008.

[19] M. Karow And E. Mengi, Matrix Polynomials with Specified Eigenvalues, preprint, arXiv:1206.6967v2, 2013.

[20] M. V. KelDysh, On the completeness of the eigenfunctions of some classes of non-selfadjoint linear operators, Russian Math. Surveys, 26 (1971), pp. 15-44.

[21] D. Kressner, A block Newton method for nonlinear eigenvalue problems, Numer. Math., 114 (2009), pp. 355-372.

[22] D. Kressner, E. Mengi, I. Nakic, and N. Truhar, Generalized eigenvalue problems with specified eigenvalues, IMA J. Numer. Anal., 34 (2014), pp. 480-501.

[23] B.-S. LiaO, Z. Bai, L.-Q. LeE, And K. Ko, Nonlinear Rayleigh-Ritz iterative method for solving large scale nonlinear eigenvalue problems, Taiwanese J. Math., 14 (2010), pp. 869-883.

[24] A. N. Malyshev, A formula for the 2-norm distance from a matrix to the set of matrices with multiple eigenvalues, Numer. Math., 83 (1999), pp. 443-454.

[25] V. Mehrmann and H. Voss, Nonlinear eigenvalue problems: A challenge for modern eigenvalue methods, GAMM Mitt. Ges. Angew. Math. Mech., 27 (2004), pp. 121-152.

[26] E. Mengi, E. A. Yildirim, And M. Kiliç, Numerical optimization of eigenvalues of Hermitian matrix functions, SIAM J. Matrix Anal. Appl., 35 (2014), pp. 699-724.

[27] R. Mennicken and M. Möller, Non-self-adjoint Boundary Eigenvalue Problems, NorthHolland Math. Stud. 192, North-Holland, Amsterdam, 2003.

[28] W. Michiels, K. Green, T. Wagenknecht, And S.-I. Niculescu, Pseudospectra and stability radii for analytic matrix functions with application to time-delay systems, Linear Algebra Appl., 418 (2006), pp. 315-335.

[29] A. RuHE, Algorithms for the nonlinear eigenvalue problem, SIAM J. Numer. Anal., 10 (1973), pp. 674-689.

[30] F. Tisseur And N. J. Higham, Structured pseudospectra for polynomial eigenvalue problems, with applications, SIAM J. Matrix Anal. Appl., 23 (2001), pp. 187-208.

[31] L. N. Trefethen and M. Embree, Spectra and Pseudospectra. The Behavior of Nonnormal Matrices and Operators, Princeton University Press, Princeton, NJ, 2005.

[32] H. Voss, Nonlinear eigenvalue problems, in Handbook of Linear Algebra, 2nd ed., L. Hogben, ed., Chapman \& Hall/CRC, Boca Raton, FL, 2013, 60.

[33] T. Wagenknecht, W. Michiels, and K. Green, Structured pseudospectra for nonlinear eigenvalue problems, J. Comput. Appl. Math., 212 (2008), pp. 245-259.

Copyright (c) by SIAM. Unauthorized reproduction of this article is prohibited. 